\title{
DS1/OsEMF1 interacts with OsARF11 to control rice architecture by regulation of brassinosteroid signaling
}

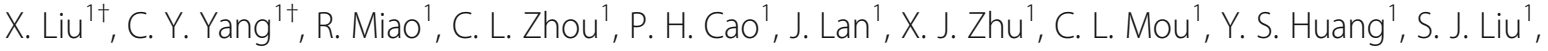 \\ Y. L. $\operatorname{Tian}^{1}$, T. L. Nguyen ${ }^{1}$, L. Jiang ${ }^{1 *}$ and J. M. $\operatorname{Wan}^{1,2^{*}}$
}

\begin{abstract}
Background: Plant height and leaf angle are important determinants of yield in rice (Oryza sativa L.). Genes involved in regulating plant height and leaf angle were identified in previous studies; however, there are many remaining unknown factors that affect rice architecture.

Results: In this study, we characterized a dwarf mutant named ds 1 with small grain size and decreased leaf angle,selected from an irradiated population of ssp. japonica variety Nanjing35. The ds 1 mutant also showed abnormal floral organs. ds 1 plants were insensitive to BL treatment and expression of genes related to BR signaling was changed. An $F_{2}$ population from a cross between ds 1 and indica cultivar 93-11 was used to fine map DS1 and to map-based clone the DS1 allele, which encoded an EMF1-like protein that acted as a transcriptional regulator. DS1 was constitutively expressed in various tissues, and especially highly expressed in young leaves, panicles and seeds. We showed that the DS1 protein interacted with auxin response factor 11 (OsARF11), a major transcriptional regulator of plant height and leaf angle, to co-regulate D61/OsBR/1 expression. These findings provide novel insights into understanding the molecular mechanisms by which DS1 integrates auxin and brassinosteroid signaling in rice.

Conclusion: The DS1 gene encoded an EMF1-like protein in rice. The ds1 mutation altered the expression of genes related to BR signaling, and ds 1 was insensitive to BL treatment. DS1 interacts with OsARF11 to co-regulate OsBR/1 expression.
\end{abstract}

Keywords: Brassinosteroids (BRs), Plant architecture, Oryza sativa, D61/OsBR/1

\section{Background}

Rice is an important food crop that feeds more than half of the world population. With an increasing global population, food security and safety are becoming serious issues. Given the limited availability of arable land and the growing human population, a future increase in rice production will be a major challenge for rice breeders (Wang et al. 2008). Plant height and leaf angle are important agronomic traits directly affecting grain yield and crop architecture, which are major objectives of crop improvement (Ikeda et al. 2013).

\footnotetext{
* Correspondence: jiangling@njau.edu.cn; wanjm@njau.edu.cn

${ }^{\dagger} X$. Liu and C. Y. Yang contributed equally to this work.

${ }^{1}$ State Key Laboratory for Crop Genetics and Germplasm Enhancement, Jiangsu Plant Gene Engineering Research Center, Nanjing Agricultural University, Nanjing 210095, China

Full list of author information is available at the end of the article
}

Plant height, an important trait in crop improvement, is related to lodging resistance, grain yield, and biomass production. Reduced plant height and associated lodging resistance are predominant strategies in crop improvement (Ayano et al. 2014). Various factors cause reduced height in plants; gibberellin (GA) and brassinosteroids (BRs) are the most widely investigated factors affecting plant height in rice. Many rice GA- and BR-related mutants show dwarf or semi-dwarf phenotypes, such as $s d 1, d 1$ and $d 61$ (Sasaki et al. 2002; Fujisawa et al. 1999; Zhang et al. 2016; Yamamuro et al. 2000). These dwarf mutants result from reduced cell numbers or cell length in stems, and analyzing more dwarf mutants may provide novel insights into the mechanisms controlling stem elongation.

Leaf angle, the degree of bending between the leaf blade and culm, is a key factor determining the plant 
architecture and grain yield (Zhao et al. 2010). Crops with erect leaves have increased photosynthetic effciency and nitrogen storage for grain flling and are suitable for dense planting (Sakamoto et al. 2006). Many genes or QTLs have been reported to control leaf angle, including D61/ OsBRI1, ILI1, LC2, ILA1, RAV6, OsARF19, and SLG (Yamamuro et al. 2000; Zhang et al. 2009; Zhao et al. 2010; Ning et al. 2011; Zhang et al. 2015b; Zhang et al. 2015a; Feng et al. 2016). Most identified rice mutants with altered leaf inclination have abnormal cell division and or expansion and altered cell wall composition at the leaf:stem joint (Zhang et al. 2009; Zhao et al. 2010). Nevertheless, BR and auxin synergistically control leaf inclination in rice (Wada et al. 1981; Hirano et al. 2017).

The synergetic effects of auxin and BRs on various physiological events have strongly suggested the interdependency of their signaling, and molecular evidence for this has been reported. For example, the auxin-inducible genes, indole-5-acetic acid (IAA5), IAA19 and SAUR-AC1, are induced by brassinolide $(\mathrm{BL})$ treatment, whereas the expression of IAA5 and IAA19 are down-regulated in the Arabidopsis BR-deficient mutant de-etiolated 2 (det2) (Nakamura et al. 2003). In addition, expression of BR biosynthetic gene DWARF4 and the BR receptor BRI1 are induced by auxin (Park et al. 2011; Sakamoto et al. 2012). BRASSINAZOLE RESISTANT 1 (BZR1) directly regulates many auxin-responsive genes (Sun et al. 2010). Nemhauser et al. (2004) found that 48 genes were co-regulated by auxin and BL (e.g. SAUR, Aux/IAA and GH3). They also found that a TGTCTC element in the auxin-responsive element (AuxRE) was enriched in genes up-regulated by both IAA and BL. These results strongly suggest that the two hormone pathways synergistically affect gene expression at the transcriptional level.

In this study, we identified a reduced BR-sensitivity mutant, ds1, showing dwarfness, smaller seed and decreased leaf angle compared to wild type. The small plant stature of the plants and decreased leaf angle resulted from reduced cell elongation. Our genetic mapping and molecular biology experiments revealed that the underlying $D S 1$, a new allele of OsEMF1, encodes an EMF1-like protein involved in the development of plant height and leaf angle by interacting with OsARF11 to co-regulate OsBRI1 expression.

\section{Results}

\section{Characterization of the $d s 1$ mutant}

To identify genetic factors regulating rice architecture, we screened our Nanjing 35 radiation mutagenesis population by isolating dwarf mutants. A mutant was identified with dwarfism and decreased leaf angles, $d s 1$ (Fig. 1a-d).

Differences between wild type and $d s 1$ mutant plants became apparent only after internode elongation when reduced height and darker leaf colour of the mutant were expressed. There was no difference between $d s 1$ and WT in young seedlings. Leaves of the mutant remained erect until maturity (Fig. 1a). Spikelet number per panicle, numbers of primary and secondary panicle branches of $d s 1$ were less than the WT (Additional file 1: Table S1). The panicles of $d s 1$ at maturity were almost erect whereas the panicles of WT clearly bend down. All internodes of $d s 1$ were shorter than those of WT (Fig. 1b-c). The dwarfism of $d s 1$ belonged to the dn type based on the classification of Yamamuro et al. (2000). Flag and second leaf angles of $d s 1$ were smaller than WT (Fig. 2a-b). These altered morphologies of $d s 1$ suggested that DS1 participated in rice growth and development.

\section{The ds 1 mutant is less sensitive to BRs}

As $d s 1$ had dwarfism, erect and dark green leaves, characteristic phenotypes of BR-related mutants, we

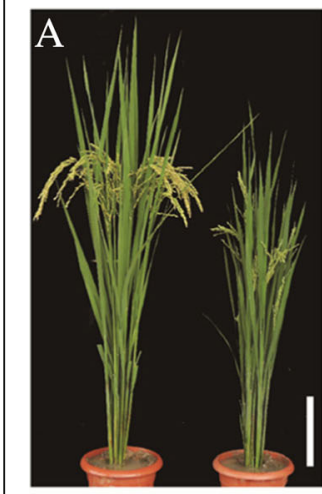

WT

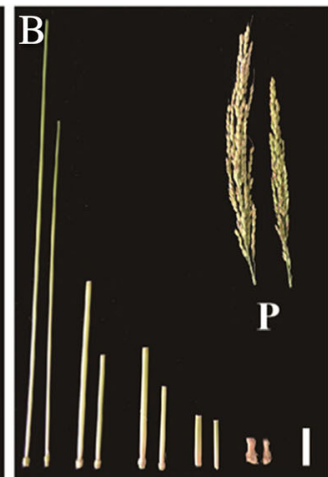

I II III IV V

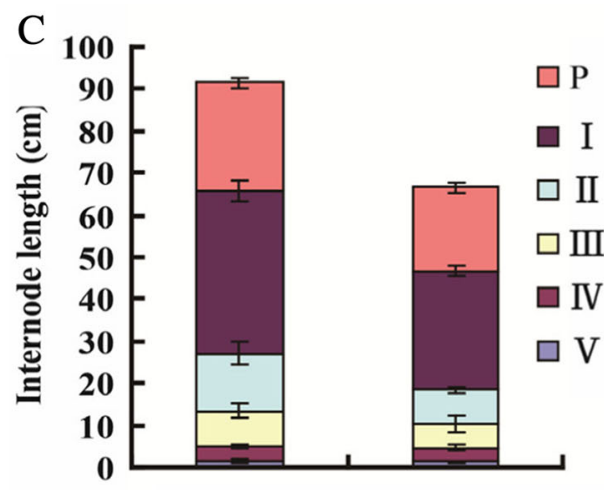

WT

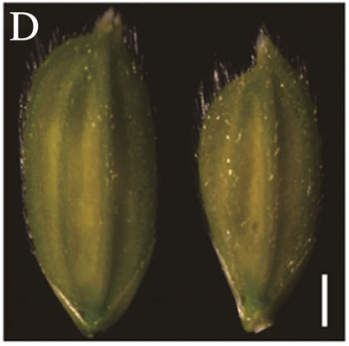

WT

Fig. 1 Phenotypic comparison of wild type and ds 1 mutant. a Gross morphology of the wild type and ds 1 plants. Scale bar, $10 \mathrm{~cm}$. b Comparison of internode and panicle lengths between WT and ds1. Scale bar, $2 \mathrm{~cm}$. c Statistical data for internode lengths described in (b). Data are presented as means \pm SD. $\mathbf{d}$ Young spikelet hulls of WT (left) and ds 1 (right) plants. Scale bar, $1 \mathrm{~mm}$ 


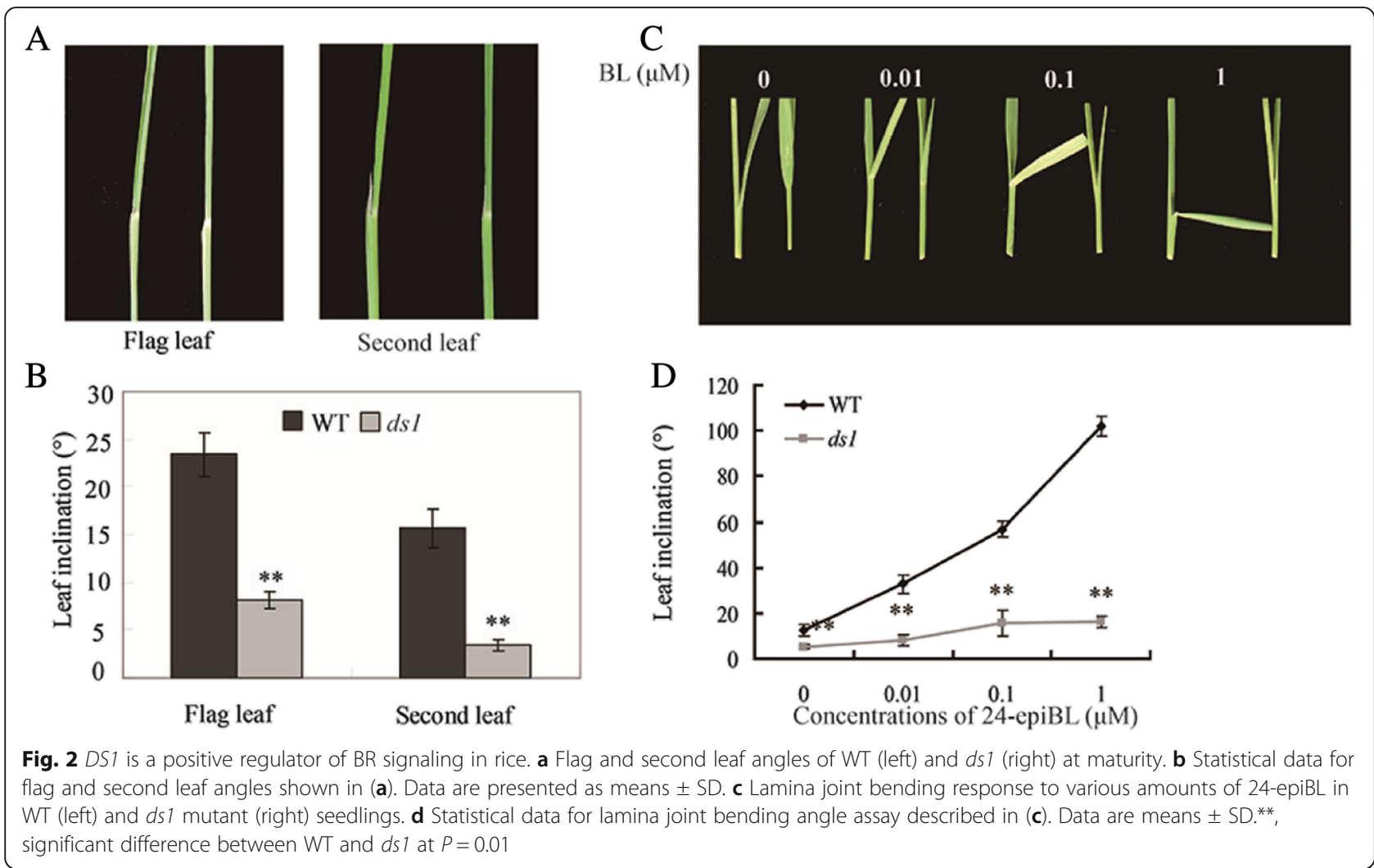

hypothesized that $D S 1$ was involved in the BR pathway. Leaf angle in rice was known to be sensitive to the concentration of $\mathrm{BL}$ or related compounds (Wada et al. 1981). We tested the sensitivity of $d s 1$ to 24-epiBL in a lamina joint bending experiment. BL treatment caused a dose-dependent lamina joint inclination in WT, whereas ds1 plants were insensitive. Treatment with BL showed an increased level leaf angle in WT plants, but little change in $d s 1$ plants (Fig. 2c-d).

\section{DS1 regulates plant height and leaf angle by promoting cell elongation}

To investigate the mutant phenotype of $d s 1$ at a cellular level we performed scanning electron microscopy (SEM) on $d s 1$ plants along with the WT (Fig. 3a-c). Dwarfism of $d s 1$ plants was mainly due to reduced cell length as measured in 2nd internodes (Fig. 3a-b). Observations on adaxial cells, which influence leaf angle, showed that those cells were also shorted in the $d s 1$ mutant (Fig. 3c). These results indicated that reduced cell length caused the dwarf phenotype of $d s 1$.

As the cell wall is the major factor restricting cell elongation, many genes involved in cell wall synthesis are reported to regulate cell elongation (Ning et al. 2011). We investigated the expression levels of cell wall synthesis-related genes in WT and the $d s 1$ mutant. Expression levels of IRX10L, CESA6, and UGA4e were significantly down-regulated in $d s 1$ compared to WT (Additional file 1: Figure S1). Consistent with the morphological observations, this indicated that the dwarfism in $d s 1$ was caused by reduced cell length. To eliminate the possibility of decreased cell size in $d s 1$, we analysed the expression levels of nine cell expansion regulators in WT and the $d s 1$ mutant (Additional file 1: Figure S1). Seven genes were significantly down-regulated in $d s 1$, indicating that cell expansion was reduced. These results further confirmed that reduced cell elongation was the cause of the phenotype of $d s 1$.

\section{Map-based cloning and functional analysis of DS1}

To determine the gene responsible for the $d s 1$ phenotype, we made a cross between $d s 1$ and the wild type. $\mathrm{F}_{1}$ plants showed the WT phenotype and the $F_{2}$ segregation ratio was consistent with that expected a single locus $\left(\chi_{3: 1}^{2}=0.98, P>0.05\right)$. These results indicated that $d s 1$ phenotype was due to a single recessive gene.

To map the DS1 locus, we next generated an $\mathrm{F}_{2}$ population from a cross between $d s 1$ and indica cultivar 9311. DS1 was roughly mapped on the short arm of chromosome 1 between SSR markers $\mathrm{N} 1-12$ and N1017. We then used 568 recessive individuals from the same $\mathrm{F}_{2}$ population and narrowed down the DS1 locus to a $80 \mathrm{~kb}$ region between markers L-3 and L-2 within BAC clones P0452F10 and P0485D09. A total of 11 

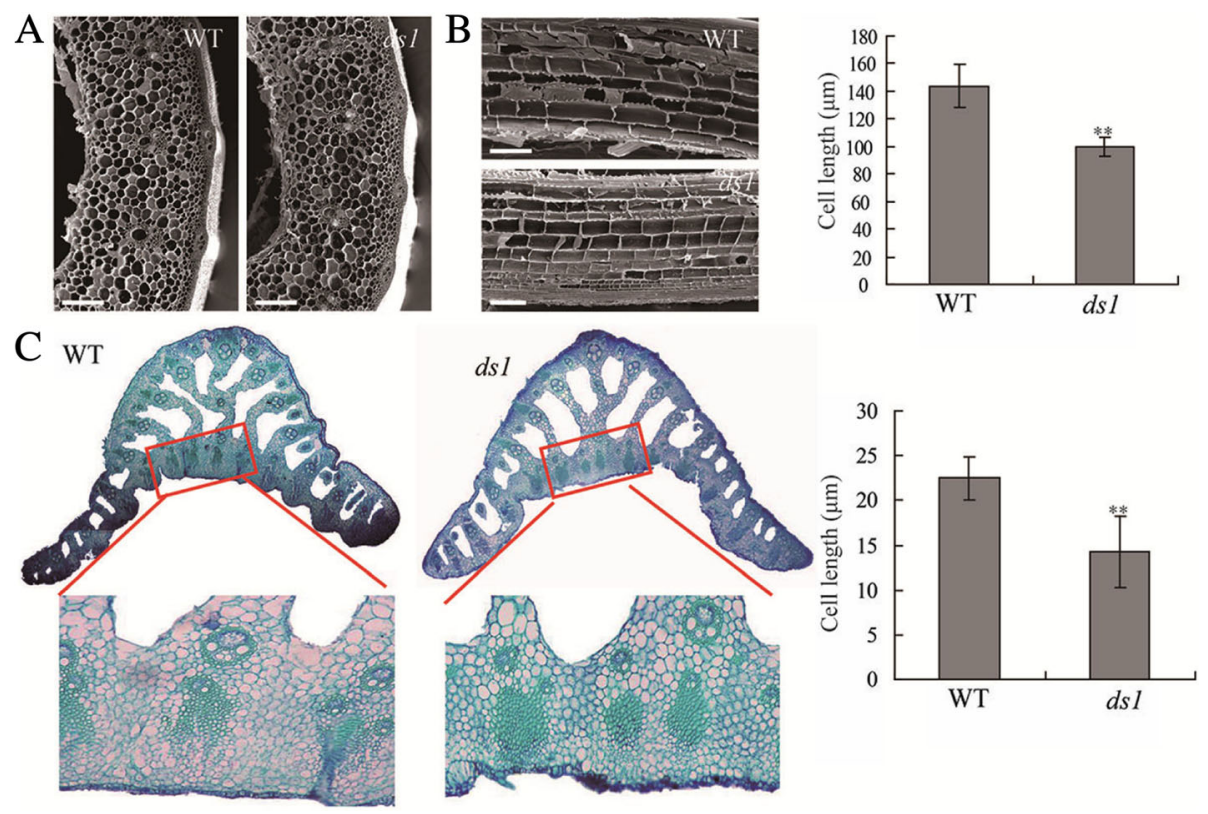

Fig. 3 Histological analysis of the 2nd internodes and lamina joints. a Transverse sections of WT and ds 1 mutant 2nd internodes. Bar, $100 \mu \mathrm{m}$. $\mathbf{b}$ Longitudinal sections of WT and ds 1 mutant 2nd internodes. Bar, $500 \mu \mathrm{m}^{* *}$ indicates significant difference at $P<0.01$. c Cross section of lamina joints of flag leaves in WT (left) and ds 1 (right). ${ }^{*}$ indicates significant difference at $P<0.01$

putative genes were localized in the $80 \mathrm{~kb}$ region according to the genome annotation of Nipponbare (http:// www.gramene.org/). On comparison of the mapped genomic region between WT and $d s 1$, we detected a single nucleotide deletion at the first exon and two nucleotide deletions in the second exon of LOC_OsO1g12890 (Fig. 4a). We identified three domains in the DS1 polypeptide, including a nuclear localization signal (NLS), an ATP/GTP binding motif, and an LXXLL motif (Fig. 4b). The LXXLL motif had been demonstrated to mediate interactions that can activate or repress transcription (Heery et al. 1997; Torchia et al. 1997; Calonje et al. 2008).

The OsEMF1 gene, annotated as the LOC_Os01g12890 gene model, encodes an EMF1-like protein, involved in H3K27me3-mediated epigenetic silencing. In Arabidopsis, EMBRYONIC FLOWER 1 (EMF1), a plant-specific protein, participated in H3K27me3-mediated silencing of target genes by acting downstream of $E M F 2$ and likely interacted with both PRC1 RING-finger proteins and PRC2 component MULTICOPY SUPRESSOR OFIRA1 (MSI1) (Aubert et al. 2001; Calonje et al. 2008). In rice, OsEMF1 plays an important role in palea development through maintaining H3K27me3-mediated epigenetic repression on OsMADS58 (Zheng et al. 2015; Yan et al. 2015). To confirm whether mutation of $D S 1$ was responsible for the $d s 1$ phenotype, a CDS fragment of 3174 bp containing the entire DS1 coding sequence and the DS1 promoter were cloned into the pCUbi1390 binary vector, and the complementation construct was then transformed into the $d s 1$ mutant. All 8 independent DS1-complementation lines rescued normal leaf angle and plant height of $d s 1$ plants (Fig. 4c). These genetic evidence and transformation results confirmed that DS1 was a newly identified OsEMF1 allele, which influenced leaf angle and plant height. Homology analysis found that amino acid sequence of DS1 had $37 \%$ similarity and 20\% homology to Arabidopsis thaliana EMF1 (AtEMF1) (Additional file 1: Figure S2).

\section{Expression pattern of DS1}

To further explore the biological functions of $D S 1$, we found that DS1 was highly expressed in the seeds,young leaves and panicles according to the Rice eFP Browser (http://bar.utoronto.ca/efprice/, Fig. 5a). Then we tested the DS1 expression pattern in various organs from WT and $d s 1$ by quantitative real-time PCR. The results showed that DS1 was highly expressed in young leaves, panicles and seeds, with the lowest expression in the roots. In comparison with the WT, the expression level was not significantly different in all tested organs of the $d s 1$ mutant (Fig. 5b). To determine whether the expression of DS1 was affected by BR, seeds were cultured on half-strength medium containing $1 \mu \mathrm{M} 24$-epiBL and expression of DS1 was determined by quantitative real-time PCR. As shown in Fig. 5c, the DS1 expression was induced by BL treatment in WT.

\section{DS1 interacts with OsARF11}

To gain a deeper insight into the molecular function of DS1, we performed a yeast two-hybrid ( $\mathrm{Y} 2 \mathrm{H})$ screening using DS1 as bait. We obtained many candidate 

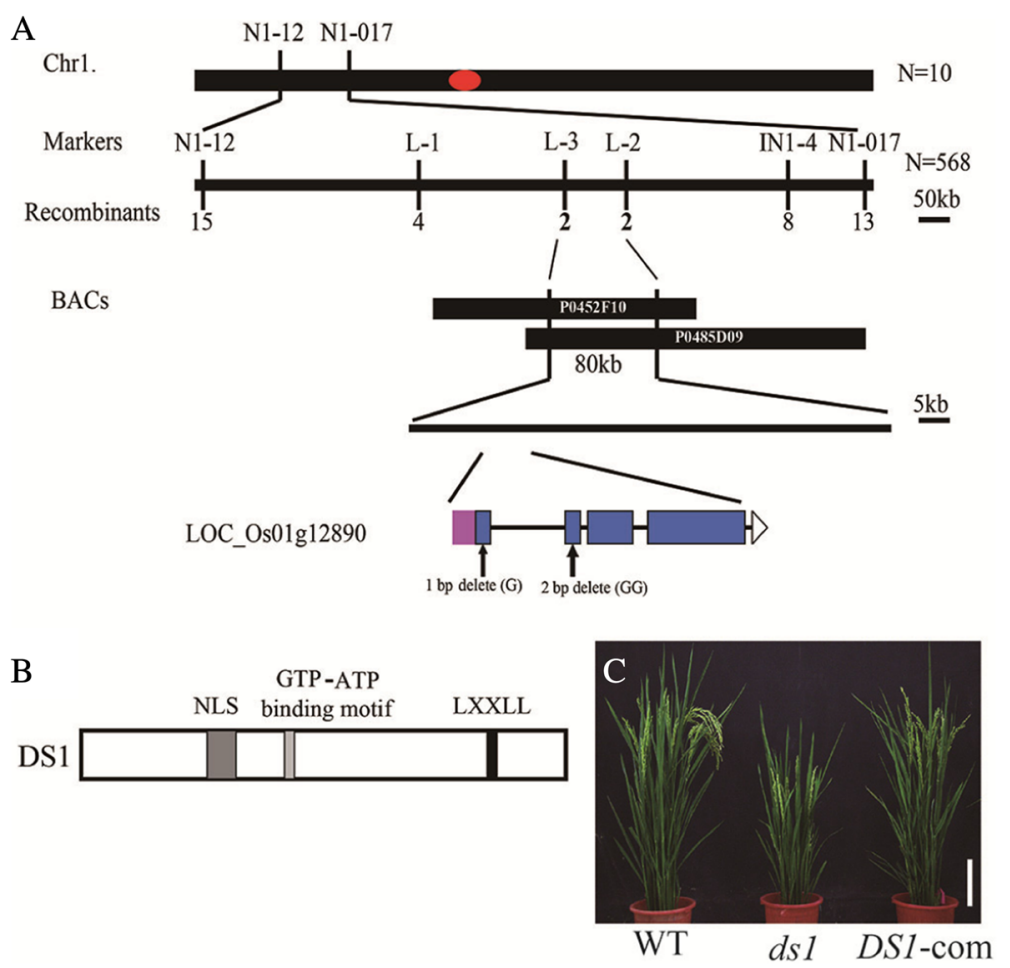

Fig. 4 Map-based cloning of DS1. a Fine mapping of the DS1 gene on chromosome 1. The DS1 locus was mapped to an $80 \mathrm{~kb}$ region that contained 11 predicted open reading frames (ORFs). b DS1 protein structure. Gray box, NLS; light gray box, GTP-ATP binding motif; black box, LXXLL motif. c Characterization of $T_{1}$ transgenic plants when ds 1 was transformed with the introduction of DS1 fragment. Bar, $10 \mathrm{~cm}$

interacting proteins, including auxin response factor 11 (OsARF11). The interaction between DS1 and OsARF11 was further confirmed using the full-length cDNA in yeast (Fig. 6a-b). Auxin response factors (ARFs), including OsARF11 and OsARF19, were reported to regulating plant height and leaf angle. Loss-of-function osarf mutants showed decreased leaf angle and dwarfism, similar to the $d s 1$ mutant. Also, OsARFs directly regulate expression of OsBRI1 by directly binding to the AuxRE element in the promoter of OsBRI1 (Shen et al. 2010; Sakamoto et al. 2013; Zhang et al. 2015a).To validate this interaction, we performed a bimolecular fluorescence complementation (BiFC) study. The N-terminal half of YFP was fused to DS1, and the C-terminal half of YFP was fused to OsARF11. Both constructs were then transiently expressed in tobacco (Nicotiana bethamiana). YFP fluorescence was observed in the nucleus, confirming that DS1 interacted with OsARF11 in planta (Fig. 6c). Taken together, these results indicated that DS1 might regulate plant height and leaf angle through interaction with OsARF11.

\section{Expression pattern and subcellular localization of OsARF11}

To verify whether the expression pattern of OsARF11 is similar to that of DS1, we found that OsARF11 was highly expressed in the shoot apical meristem and young panicles according to the Rice eFP Browser (http://bar.utoronto.ca/efprice/, Fig. 7a). We employed a quantitative RT-PCR (qRT-PCR) assay to examine OsARF11 expression in wild-type plants. Real time qPCR analysis showed that OsARF11 transcripts accumulated in all examined tissues, including sheaths, young panicles, roots, stems and leaves (Fig. 7b). These results indicated that OsARF11 showed a very similar expression pattern to DS1. We further, found that OsARF11 expression was induced by auxin treatment (Fig. 7c), suggesting that its function might be regulated by auxin. We next examined the subcellular localization of OsARF11 protein. A construct harboring a OsARF11-GFP fusion was introduced into tobacco epidermal cells by Agrobacterium. Fluorescent signals of OsARF11-GFP fusion protein were observed exclusively in the nucleus of tobacco cells (Fig. 7d), indicating that OsARF11 is a nuclear protein, consistent with the function of OsARF11.

\section{DS1 and OsARF11 co-regulate D61/OsBRI1 expression}

As shown in Fig. 2c, the ds1 mutant was insensitive to $\mathrm{BL}$ treatment. We investigated the effect of DS1 on the expression of BR-related genes and the results showed that disruption of DS1 led to significantly increased expression of D2 and DWARF4, two important 


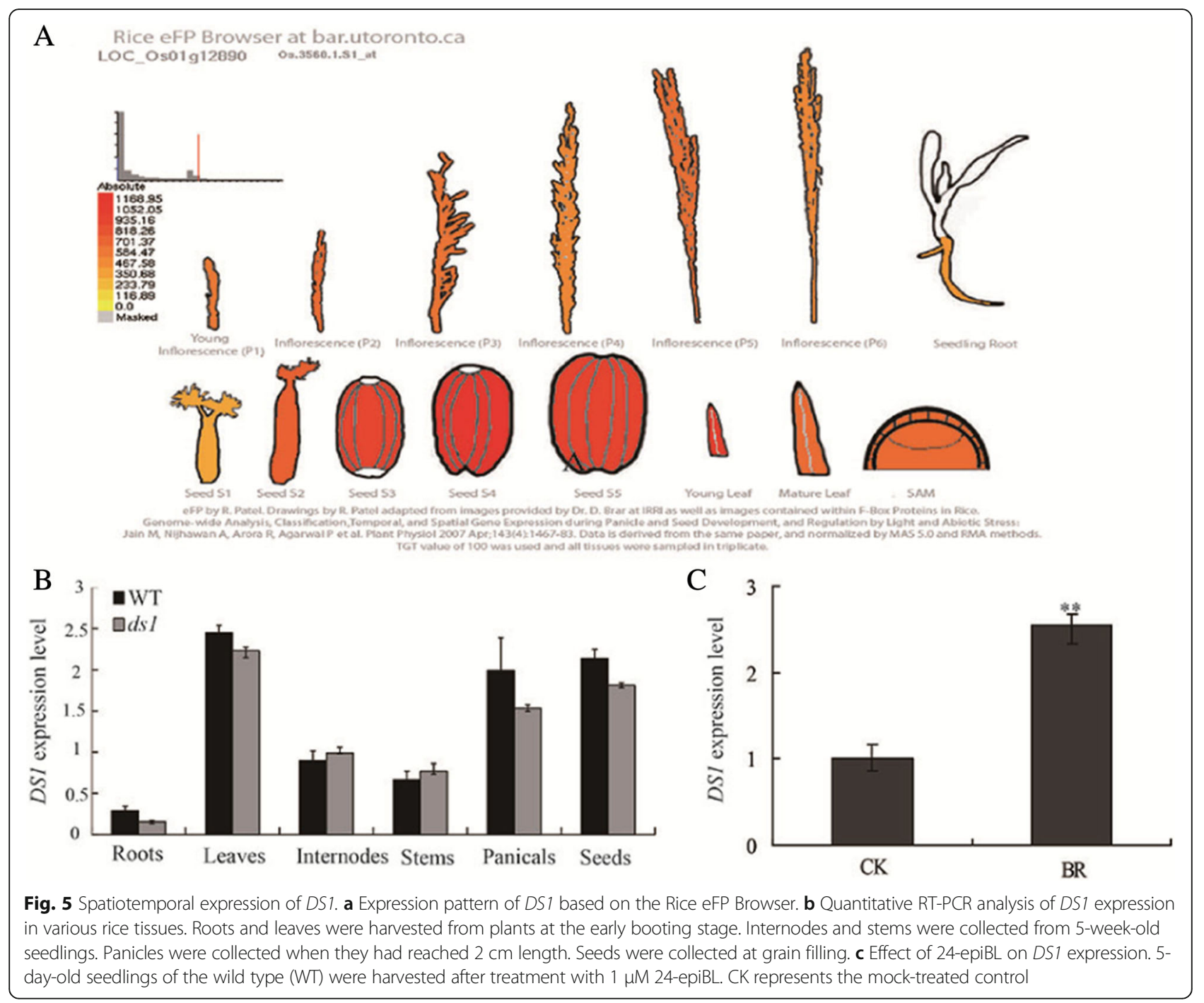

brassinosteroid biosynthesis rate-limiting enzymes (Hong et al. 2003; Sakamoto et al. 2006). By contrast, DS1 had large effects on expression of BR signal genes, including D61/OsBRI1, OsBZR1, OsBU1 and XTR1 (Fig. 8). This indicated that $D S 1$ could be involved in BR signaling.

Previous studies showed that OsARF11 promotes plant growth and leaf angle by specific binding to an auxin response element (AuxRE) in the promoter of OsBRI1 (Sakamoto et al. 2013), and that OsARF19 binds to an AuxRE element in the promoters of OsBRI1 and OsGH35 (Zhang et al. 2015a). We analyzed the promoters of four BR signal genes (OsBRI1, OsBZR1, OsBU1 and XTR1), and found that only the promoter of OsBRI1 contained the auxin response element. To confirm the binding of DS1 or OsARF11 to the OsBRI1 promoter, we performed yeast one hybrid assays using in vitro-expressed DS1 and OsARF11. As shown in Fig. 9a, OsARF11 bound to the OsBRI1 promoter but DS1 failed to bind the promoter of OsBRI1.
To examine the regulation of DS1 and OsARF11 on the expression of its target gene OsBRI1, we performed transient expression assays using $\sim 2 \mathrm{~kb}$ of the OsBRI1 promoter fused to the LUC gene as a reporter. Effector constructs for OsARF11 were expressed under control of the $35 \mathrm{~S}$ promoter and transfected together with the reporter construct into rice protoplasts. Higher LUC activity was detected when OsARF11 protein was transfected with the reporter construct compared with the internal control (Fig. 9b). These findings strongly support the hypothesis that DS1 and OsARF11 coregulate OsBRI1 in controlling rice plant architecture.

\section{Discussion}

Plant architecture is a major factor underlying grain yield and determination of planting density and photosynthesis efficiency in rice. The molecular mechanisms of plant architecture have been extensively studied and many critical genes have been identified. Most of these 


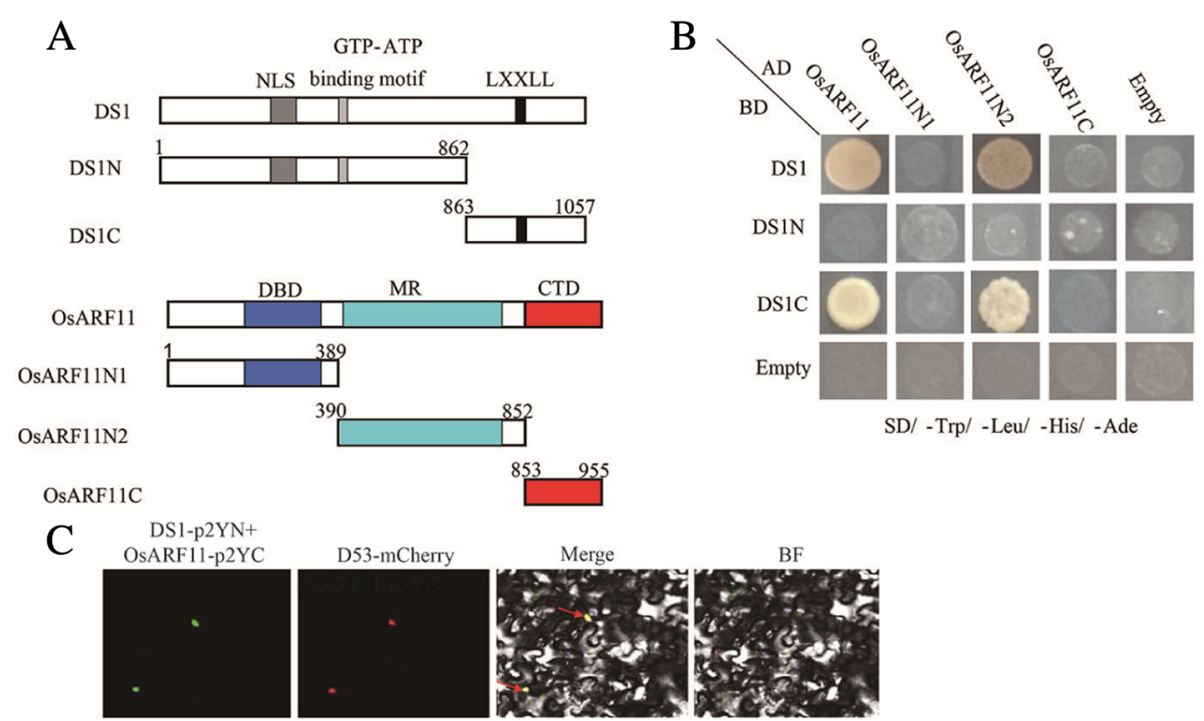

Fig. 6 DS1 physically interacts with OSARF11. a Sketches showing the domain structures of DS1 and OsARF11 and various deletions. b Yeast twohybrid assays showing the interactions between DS1, OsARF11, and their derivatives. Transformed yeast cells were grown on SD/-Trp/-Leu/-His/ -Ade. c BiFC assay showing DS1-YFPN and OsARF11-YFPC interaction to form a functional YFP in the nucleus

genes are involved in the Auxin and BR pathways. Auxin and BR signaling share many transcriptional target genes in regulating rice plant architecture (Yin et al. 2002; Nemhauser et al. 2004), suggesting that interactions between TFs involved in auxin and BR signaling might act as the points of auxin-BR crosstalk, but only a few examples have been reported. One is the response of SAUR15 to auxin and BR. SAUR15 expression depends on the simultaneous interaction of BES1 and ARF5 within the promoter of the SAUR15, gene that produces a hormone with an Up at Dawn (HUD)-type E-box and AuxRE cis elements (Walcher and Nemhauser 2012). Another example is that BZR1 and ARF6 were shown to interact and regulate a large number of common target genes (Oh et al. 2014).

In this study, we characterized mutant $d s 1$, which showed dwarfing and a reduced leaf angle compared to the WT. Through map-based cloning and genetic transformation, DS1 was identified as a new allele of $O s E M F 1$. In rice, OsEMF1 functions in influencing the level of the H3K27me3 at the OsMADS58 locus, and repressing the expression of OsMADS58 to control palea development (Zheng et al. 2015; Yan et al. 2015). ds1 became less sensitive to BL treatment relative to the wild type in a lamina joint inclination test. We found that the expression level of OsBRI1 was down-regulated 5 times in the $d s 1$ mutant. OsEMF1 inhibits the expression of target genes by mediating $\mathrm{H} 3 \mathrm{~K} 27 \mathrm{me} 3$, which is not consistent the down-regulation of OsBRI1 expression in the ds1 mutant. DS1 may influence the expression of OsBRI1 through histone methylation or other unknown mechanisms. We demonstrated that DS1 physically interacted with OsARF11 to positively co-regulate expression of OsBRI1. Indeed, DS1 and OSARF11 showed similar expression profiles.

Following the initial discovery of the effect of auxin-BR crosstalk on lamina joint bending, several genes have now been reported to be involved (Luo et al. 2016). For instance, $L P A 1$ negatively regulates lamina joint bending by inhibiting BR signaling and increasing the expression of auxin transporters (Liu et al. 2016). In a recent study, the SMOS1-SMOS2/DLT complex positively regulated expression of OsPHI-1, and treatment of smos mutants with auxin and BR still showed a synergistic effect on lamina joint bending (Hirano et al. 2017). In the current study, we showed that DS1-OsARF11 is invoved in auxin-BR crosstalk in rice. It is plausible that lamina joint bending is determined by a transcriptional network consisting of DS1-OsARF11, SMOS1-SMOS2/ DLT, LPA1, and other factors regulated by auxin and BR signaling.

In high-density plantings, characteristic semi-dwarf phenotypes with erect leaves are ideal phenotypes for improving grain yield ( $\mathrm{Lu}$ et al. 2013; Wu et al. 2008). Further, identification of other downstream genes of $D S 1 / O s E M F 1$ will be needed for fully understanding the network regulating plant growth and development. In addition, the BR signaling pathway is necessary for auxin function in controlling leaf angle, indicating that the roles of auxin and BR are interdependent. Our study uncovers a previously unknown regulatory factor in plant architecture and the coordinating network of auxin and 


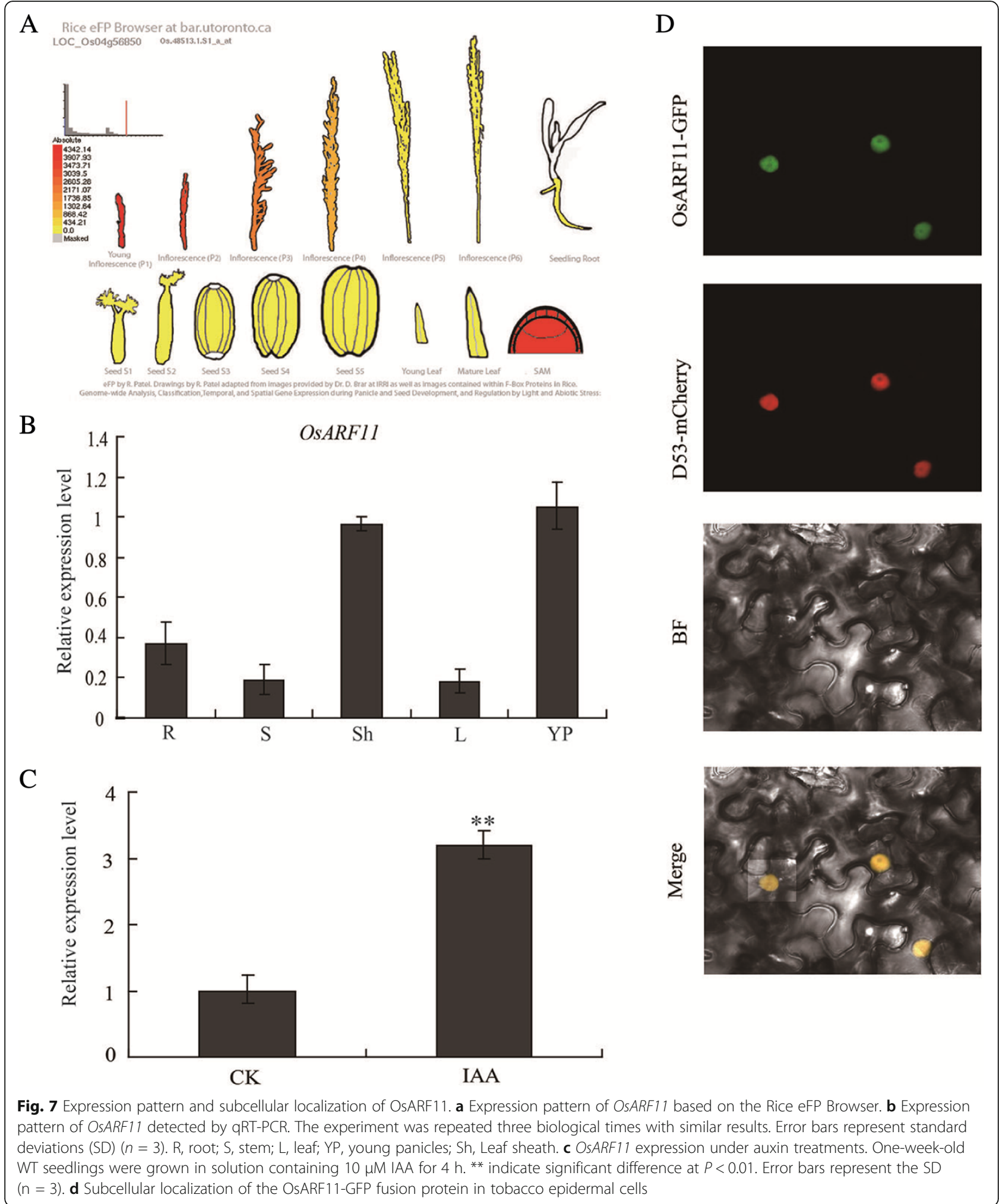



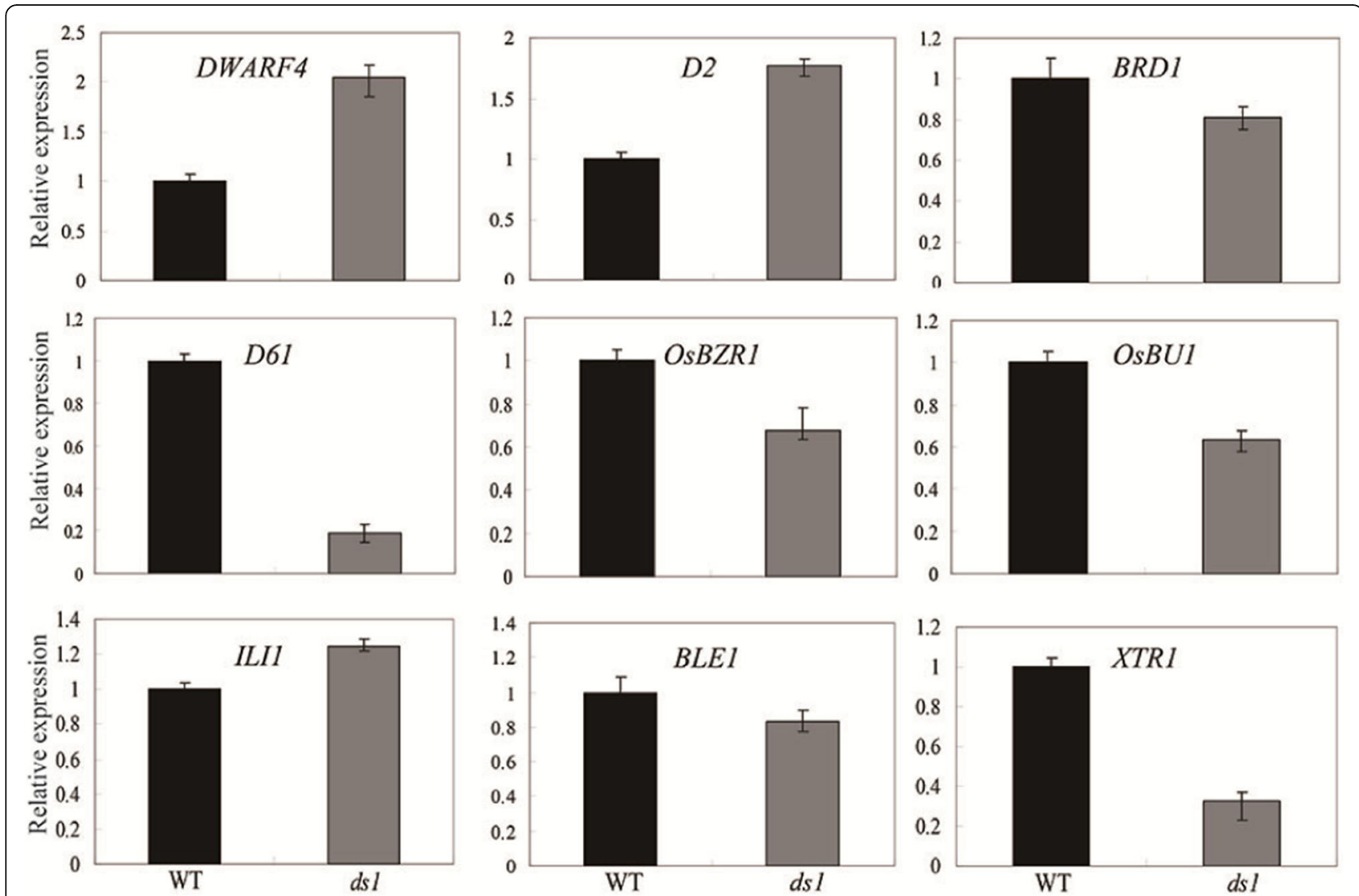

Fig. 8 Expression of BR biosynthesis and signal transduction genes. Real-time PCR analysis of BR biosynthesis and signal transduction genes in the wild type and $d s 1$ seedling at 15 days after germination. Data are presented as means $\pm S D(n=3)$

BR signaling. These findings might be useful for manipulating optimal rice architectures.

\section{Conclusions}

In this study, we found that DS1/OsEMF1 functions in BR signaling by interacting with OsARF11. The underlying mechanisms might be useful for engineering rice architecture to increase rice yield.

\section{Methods}

\section{Plant material and growth conditions}

The $d s 1$ mutant obtained from an irradiated population of japonica variety Nanjing 35 was stably inherited. The mutant was self-pollinated for several generations until shown to be heritable. Crosses were made between the $d s 1$ mutant and variety Nanjing 35 for genetic analysis. 93-11 (indica) was employed as a control in mapping of DS1. Pre-germinated seeds were sown in nursery beds in the field and the 1-month-old seedlings were transplanted into a paddy field in Nanjing.

\section{Trait measurement}

The grain length, width, and thickness were measured by an electronic digital display vernier caliper and fully filled grains were used for measuring the 1000-grain weight. The plant height, primary and secondary branch number, panicle length, and grain number per panicle were obtained from the measurement of the main stem. Numbers of tillers per plant were recorded at the time of harvest. Three biological repeats were measured for each sample.

\section{Histological analysis}

The second internodes and lamina joints of the flag leaves were harvested at heading date, respectively. All samples fixed in glutaric dialdehyde for $48 \mathrm{~h}$. The fixed internode samples were soaked in $2 \%(w / v) \mathrm{OsO}_{4}$ for $2 \mathrm{~h}$, dehydrated in a graded ethanol series $(70,80,90$, 100 , and $100 \%$ ), cleared in a xylene series, and then sputter coated with platinum. Second internode sections were observed by SEM (HITACHI, S-3000 N). Fixed lamina joints were embedded in paraplast. After sectioning, $10 \mu \mathrm{m}$ thick sections were dewaxed with xylene, rehydrated, stained with $1 \%$ toluidine blue, and observed 


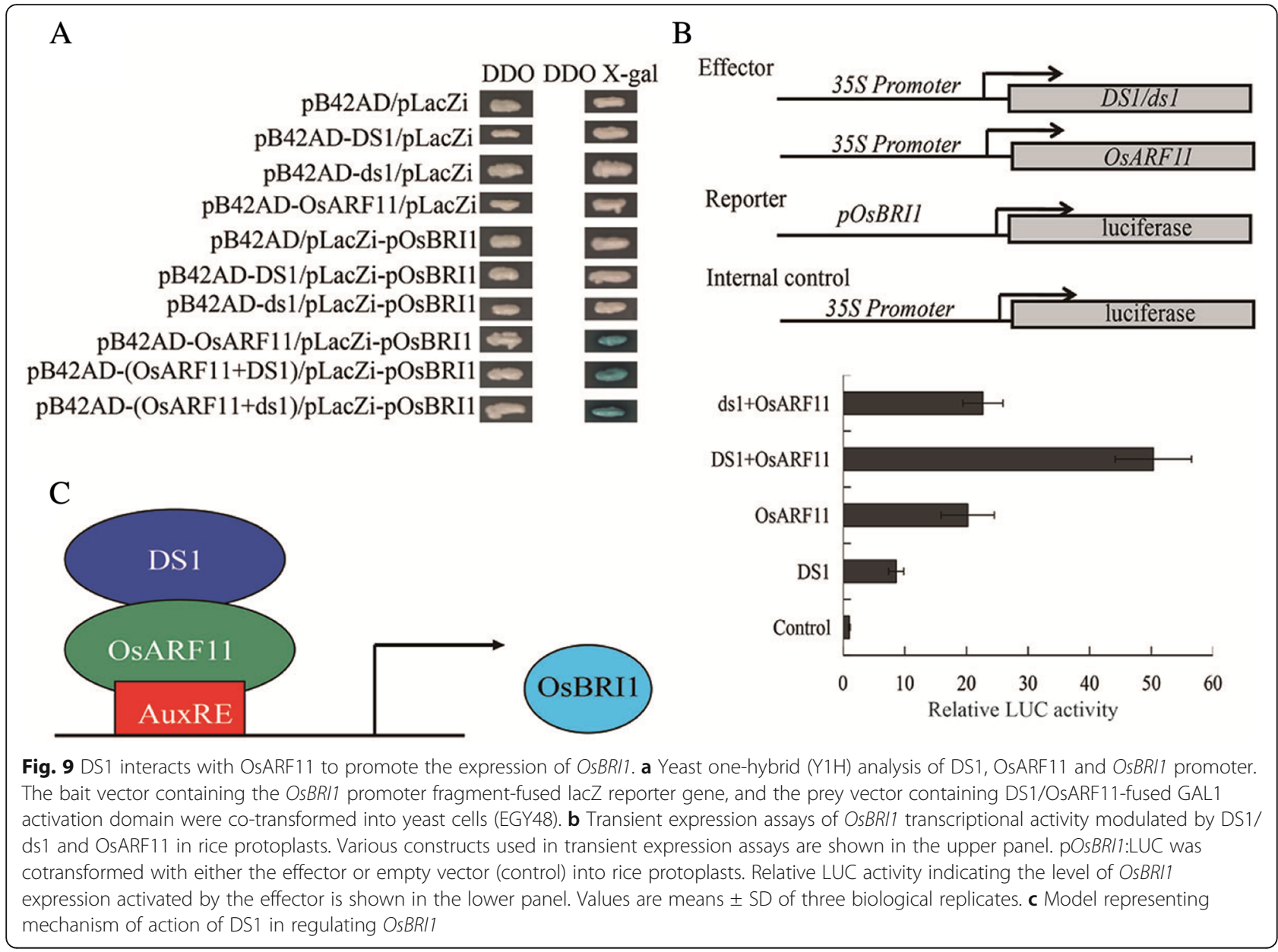

with a Leica DM5000B microscope. Cell lengths of each organ were measured with IMAGEJ software.

\section{Lamina joint bending assay}

Brassinolide (BL) was dissolved in ethanol. For BL treatment, sterile water was added to reach final concentrations of $0.01,0.1$, and $1 \mu \mathrm{M}$. Germinated seedlings of WT and $d s 1$ were grown in the light for $10 \mathrm{~d}$ at $30{ }^{\circ} \mathrm{C}$. Lamina joint segments were floated on distilled water for $72 \mathrm{~h}$ containing various concentrations of brassinolide in the light. The lamina joint bending angles were then photographed and measured. Twenty plants were used for each treatment.

\section{Genetic analysis and map-based cloning of DS1}

Populations for genetic analysis and mapping were from crosses between $d s 1$ and japonica cv. Nanjing 35 and indica cv. 93-11. For mapping of DS1, $7680 \mathrm{~F}_{2}$ plants were generated from a cross between $d s 1$ mutant and indica cv. 93-11, and 568 individuals with the clear $d s 1$ phenotypes were selected for mapping. Additional molecular markers were designed from comparative sequences of
Nipponbare and 93-11. Molecular markers chosen for mapping are listed in Additional file 1: Table S2. The PCR procedure was: $95^{\circ} \mathrm{C}$ for $5 \mathrm{~min}$, followed by 33 cycles of $95^{\circ} \mathrm{C}$ for $30 \mathrm{~s}$, annealing for $30 \mathrm{~s}, 72{ }^{\circ} \mathrm{C}$ for $40 \mathrm{~s}$ and a final elongation step at $72{ }^{\circ} \mathrm{C}$ for $5 \mathrm{~min}$. All the populations were grown in the field at Nanjing in Jiangsu province, and Lingshui County in Hainan province.

\section{Complementation of the $d s 1$ mutant}

To test whether the mutation in LOC_Os01g12890 was responsible for $d s 1$, an $\sim 2 \mathrm{~kb}$ promoter and full length cDNA of DS1 were cloned into the pCUbi1390 binary vector (primer sequences are listed in Additional file 1: Table S3). The resulting binary plasmid was then introduced into Agrobacterium tumefaciens strain EHA105. Calli induced from homogeneous $d s 1$ seeds were used for transformation. The transgenic plants were grown in a glasshouse to observe the phenotypes.

\section{RNA extraction and qRT-PCR analysis}

Total RNAs were isolated from various plant organs using Trizol reagent (Invitrogen) according to the 
manufacturer's instructions. First strand cDNAs were synthesized from $2 \mu \mathrm{g}$ total RNA using a PrimeScript 1st Strand cDNA Synthesis Kit (Takara, Dalian).

For qRT-PCR, SYBR Premix Ex TaqTM kit (Takara) was added to the reaction system and run on an $\mathrm{ABI}$ Prism 7500 real-time PCR system and the OsActin gene was used as an internal control. The relative expression level was calculated by $2^{-\Delta \Delta C t}$. The primers used for BR-related genes and cell wall synthesis-related genes are listed in Additional file 1: Table S3. The PCR procedure was: $95{ }^{\circ} \mathrm{C}$ for $30 \mathrm{~s}$, followed by 40 cycles of $95{ }^{\circ} \mathrm{C}$ for $5 \mathrm{~s}, 60^{\circ} \mathrm{C}$ for $34 \mathrm{~s}$.

\section{Yeast two-hybrid assay}

For yeast two-hybrid analysis, various fragments were cloned into pGBKT7 and pGADT7 to construct $\mathrm{BD}-\mathrm{DS} 1 / \mathrm{N} / \mathrm{C} /$ and $\mathrm{AD}-\mathrm{OsARF} 11 / \mathrm{N} 1 / \mathrm{N} 2 / \mathrm{C} /$, respectively. The reported gene assay was performed following the manufacturer's instructions (Clontech). Yeast transformation and screening procedures were performed according to the manufacturer's instructions (Clontech). The yeast cells were transformed with the rice cDNA library constructed from young panicles of the japonica cultivar "Nipponbare". Positive transformants were screened for growth on SD/-Trp/-Leu/ -His/-Ade. The yeast clones were cultured and sequenced. The primers used for yeast two-hybrid analysis were listed in Additional file 1: Table S3.

\section{BiFC assay}

The full-length cDNA of DS1 was cloned into the p2YN vector to construct the DS1-YFP ${ }^{\mathrm{N}}$ fusion protein. OsARF11 was cloned into the p2YC vector to produce an OsARF11-YFP ${ }^{C}$ fusion protein, (primer sequences are listed in Additional file 1: Table S3). BiFC analyses were performed in tobacco, as described previously (Waadt and Kudla 2008). The primers used for BiFC assay are listed in Additional file 1: Table S3.

\section{Transient expression and yeast one-hybrid assays}

To generate the pOsBRI1:LUC reporter construct, $\sim 2 \mathrm{~kb}$ of the OsBRI1 promoter was cloned into pGreenII-0800-LUC. OsARF11 and DS1 full length cDNA were cloned into the modified pAN580 binary vector, respectively. The construct vectors were co-transformed into rice protoplasts. Rice protoplasts were prepared, transfected, and cultured as previously described (Wang et al. 2016). The luciferase activity assay was calculated following the manufacturer's instructions (Promega) and the data presented are the averages of three biological replicates.

For yeast one-hybrid $(\mathrm{Y} 1 \mathrm{H})$ assays, the full length cDNA of DS1, OsARF11 and promoter of OsBRI1 were amplified using gene-specifc primers (Additional file 1: Table S3), and the amplifed products were cloned into the $\mathrm{pB} 42 \mathrm{AD}$ vector and reporter plasmid $\mathrm{pLacZi}$, respectively. Plasmids were co-transformed into yeast strain EGY48 according to the manufacturer's manual (Clontech).

\section{Additional file}

Additional file 1: Table S1 Comparison of agronomic traits between WT and ds1. Table S2 Primers used in mapping. Table S3 Primers used in real-time PCR and vector construction. Figure $\mathbf{S 1}$ Relative expression levels of cell wall synthesis-related genes (CESA6, IRX10L, GT8, UGA4e, and CSLF6) and cell expansion-related genes, Exp1, ExpA8, ExpA10, ExpA17, ExpA30, ExpB2, ExpB3, ExpB5, and ExpB12. Figure S2 Alignment amino acid sequences of DS1 and AtEMF1. (DOC $1088 \mathrm{~kb}$ )

\section{Abbreviations}

BiFC: Bimolecular fluorescence complementation; GFP: Green fluorescent protein; OsARF11: Auxin response factor 11; qRT-PCR: Quantitative real-time PCR; SEM: Scanning Electron Microscope; WT: Wild type; Y1H: Yeast onehybrid

\section{Acknowledgements \\ We are thankful to Dr. Chunming Wang for critical comments on the manuscript.}

\section{Funding}

This research was supported by the Key Laboratory of Biology, Genetics and Breeding of Japonica Rice in Mid-lower Yangtze River, Ministry of Agriculture, P. R. China, and Jiangsu Collaborative Innovation Center for Modern Crop Production, and grants from The National Key Research and Development Program of China (2016YFD0100101-08), Jiangsu Science and Technology Development Program (BE2017368), and Agricultural Science and Technology Innovation Fund project of Jiangsu Province (CX(16)1029).

\section{Availability of data and materials}

All data supporting the conclusions of this article are provided within the article (and its additional files).

Authors' contributions

$L X, Y C Y, J L$ and WJM conceived and designed the experiments. $L X, Y C Y, M R$, $L J, C P H, Z C L, Z X J, M C L, H Y S$ and NTL performed the experiments and analyzed the data. LSJ and TYL were responsible for material plant and field management. LX and YCY wrote the manuscript. JL and WJM revised the manuscript. All authors read and approved the manuscript.

\section{Author information}

Correspondence and requests for materials should be addressed to Ling Jiang (jiangling@njau.edu.cn) and Jianmin Wan (wanjm@njau.edu.cn).

Ethics approval and consent to participate Not applicable.

Consent for publication

Not applicable.

\section{Competing interests}

The authors declare that they have no competing interests.

\section{Publisher's Note}

Springer Nature remains neutral with regard to jurisdictional claims in published maps and institutional affiliations.

\section{Author details}

'State Key Laboratory for Crop Genetics and Germplasm Enhancement, Jiangsu Plant Gene Engineering Research Center, Nanjing Agricultural University, Nanjing 210095, China. ${ }^{2}$ National Key Facility for Crop Gene Resources and Genetic Improvement, Institute of Crop Science, Chinese Academy of Agricultural Sciences, Beijing 100081, China. 
Received: 6 June 2018 Accepted: 27 July 2018

\section{Published online: 06 August 2018}

\section{References}

Aubert D, Chen L, Moon YH, Martin D, Castle LA, Yang CH, Sung ZR (2001) EMF1, a novel protein involved in the control of shoot architecture and flowering in Arabidopsis. Plant Cell 13:1865-1875

Ayano M, Kani T, Kojima M, Sakakibara H, Kitaoka T, Kuroha T, Angeles-Shim RB, Kitano H, Nagai K, Ashikari M (2014) Gibberellin biosynthesis and signal transduction is essential for internode elongation in Deepwater rice. Plant Cell Environ 37:2313-2324

Calonje M, Sanchez R, Chen L, Sung ZR (2008) EMBRYONIC FLOWER1 participates in polycomb group -mediated $A G$ gene silencing in Arabidopsis. Plant Cell 20: $277-291$

Feng ZM, Wu C, Wang C, Roh J, Zhang L, Chen J, Zhang S, Zhang H, Yang C, Hu $J$ et al (2016) SLG controls grain size and leaf angle by modulating brassinosteroid homeostasis in rice. J Exp Bot 67:4241-4253

Fujisawa Y, Kato T, Ohki S, Ishikawa A, Kitano H, Sasaki T, Asahi T, Iwasaki Y (1999) Suppression of the heterotrimeric $\mathrm{G}$ protein causes abnormal morphology, including dwarfism, in rice. Proc Natl Acad Sci U S A 96:7575-7580

Heery DM, Kalkhoven E, Hoare S, Parker MG (1997) A signature motif in transcriptional co-activators mediates binding to nuclear receptors. Nature 387:733-736

Hirano K, Yoshida H, Aya K, Kawamura M, Hayashi M, Hobo T, Sato-Izawa K, Kitano H, Ueguchi-Tanaka M, Matsuoka M et al (2017) SMALL ORGAN SIZE 1 and SMALL ORGAN SIZE 2/ DWARF and LOW-TILLERING form a complex to integrate auxin and brassinosteroid signalling in rice. Mol Plant 10:590-604

Hong Z, Ueguchi-Tanaka M, Umemura K, Uozu S, Fujioka S, Takatsuto S, Yoshida S, Ashikari M, Kitano H, Matsuoka M (2003) A rice brassinosteroid-deficient mutant, ebisu dwarf (d2), is caused by a loss of function of a new member of cytochrome P450. Plant Cell 15:2900-2910

Ikeda M, Miura K, Aya K, Kitano H, Matsuoka M (2013) Genes offering the potential for designing yield-related traits in rice. Curr Opin Plant Biol 16:213-220

Liu JM, Park SJ, Huang J, Lee EJ, XuanYH JBI, Kumar V, Priatama RA, Raj KV, Kim SH, Min MK, Cho JH, Kim TH, Chandran AK, Jung KH, Takatsuto S, Fujioka S, Han CD (2016) Loose plant Architecture1 (LPA1) determines lamina joint bending by suppressing auxin signaling that interacts with $\mathrm{C}-22-$ hydroxylated and 6-deoxo brassinosteroids in rice. J Exp Bot 67:1883-1895

Lu Z, Yu H, Xiong G, Wang J, Jiao Y, Liu G, Jing Y, Meng X, Hu X, Qian Q, Fu X, Wang Y, Li J (2013) Genome-wide binding analysis of the transcription activator ideal plant architecture 1 reveals a complex network regulating rice plant architecture. Plant Cell 25:3743-3759

Luo X, Zheng J, Huang R, Huang Y, Wang H, Jiang L, Fang X (2016) Phytohormones signaling and crosstalk regulating leaf angle in rice. Plant Cell Rep 35:2423-2433

Nakamura A, Higuchi K, Goda H, Fujiwara MT, Sawa S, Koshiba T, Shimada Y, Yoshida S (2003) Brassinolide induces IAA5, IAA19, and DR5, a synthetic auxin response element in Arabidopsis, implying a cross talk point of brassinosteroid and auxin signaling. Plant Physiol 133:1843-1853

Nemhauser JL, Mockler TC, Chory J (2004) Interdependency of brassinosteroid and auxin signaling in Arabidopsis. PLoS Biol 2:e258

Ning J, Zhang B, Wang N, Zhou Y, Xiong L (2011) Increased leaf angle 1, a Raf-like MAPKKK that interacts with a nuclear protein family, regulates mechanical tissue formation in the lamina joint of rice. Plant Cell 23:4334-4347

Oh E, Zhu JY, Bai MY, Arenhart RA, Sun Y, Wang ZY (2014) Cell elongation is regulated through a central circuit of interacting transcription factors in the Arabidopsis hypocotyl. Elife 3:e03031

Park T, Cho H, Hwang I, Choe S (2011) Auxin stimulates DWARF4 expression and brassinosteroid biosynthesis in Arabidopsis. Plant J 66:564-578

Sakamoto T, Morinaka Y, Inukai Y, Kitano H, Fujioka S (2013) Auxin signal transcription factor regulates expression of brassinosteroid receptor gene in rice. Plant J 73:676-688

Sakamoto T, Morinaka Y, Ohnishi T, Sunohara H, Fujioka S, Ueguchi-Tanaka M, Mizutani M, Sakata K, Takatsuto S, Yoshida S, Tanaka H, Kitano H, Matsuoka M (2006) Erect leaves caused by brassinosteroid deficiency increase biomass production and grain yield in rice. Nat Biotechnol 24:105-109

Sakamoto T, Ohnishi T, Fujioka S, Watanabe B, Mizutani M (2012) Rice CYP90D2 and CYP90D3 catalyze C-23 hydroxylation of brassinosteroids in vitro. Plant Physiol Biochem 58:220-226
Sasaki A, Ashikari M, Ueguchi-Tanaka M, Itoh H, Nishimura A, Swapan D, Ishiyama K, Saito T, Kobayashi M, Khush GS, Kitano H, Matsuoka M (2002) Green revolution: a mutant gibberellin-synthesis gene in rice. Nature 416:701-702

Shen C, Wang S, Bai Y, Wu Y, Zhang S, Chen M, Guilfoyle TJ, Wu P, Qi Y (2010) Functional analysis of the structural domain of ARF proteins in rice (Oryza sativa L.). J Exp Bot 61:3971-3981

Sun Y, Yang Y, Yuan Z, Muller JL, Yu C, Xu Y, Shao X, Li X, Decker EL, Reski $R$, Huang $H$ (2010) Overexpression of the Arabidopsis gene UPRIGHT ROSETTE reveals a homeostatic control for indole-3-acetic acid. Plant Physiol 153:1311-1320

Torchia J, Rose DW, Inostroza J, Kamei Y, Westin S, Glass CK, Rosenfeld MG (1997) The transcriptional coactivator $\mathrm{p} / \mathrm{CIP}$ binds CBP and mediates nuclearreceptor function. Nature 387:677-684

Waadt R, Kudla J (2008) In planta visualization of protein interactions using bimolecular fluorescence complementation (BiFC). CSH protoc 2008:t4995

Wada K, Marumo S, Ikekawa N, Morisaki M, Mori K (1981) Brassinolide and homobrassinolide promotion of lamina inclination of rice seedlings. Plant Cell Physiol 22:323-325

Walcher CL, Nemhauser JL (2012) Bipartite promoter element required for auxin response. Plant Physiol 158:273-282

Wang L, Xu Y, Zhang C, Ma Q, Joo SH, Kim SK, Xu Z, Chong K (2008) OsLIC, a novel $\mathrm{CCCH}$-type zinc finger protein with transcription activation, mediates rice architecture via brassinosteroids signaling. PLoS One 3:e3521

Wang Y, Wang C, Zheng M, Lyu J, Xu Y, Li X, Niu M, Long W, Wang D, Wang H, Terzaghi W, Wang Y, Wan JM (2016) WHITE PANICLE1, a val-tRNA synthetase regulating chloroplast ribosome biogenesis in rice, is essential for early chloroplast development. Plant Physiol 170:2110-2123

Wu CY, Trieu A, Radhakrishnan P, Kwok SF, Harris S, Zhang K, Wang J, Wan JM, Zhai H, Takatsuto S, Matsumoto S, Fujioka S, Feldmann KA, Pennell RI (2008) Brassinosteroids regulate grain filling in rice. Plant Cell 20:2130-2145

Yamamuro C, Ihara Y, Wu X, Noguchi T, Fujioka S, Takatsuto S, Ashikari M, Kitano H, Matsuoka M (2000) Loss of function of a rice brassinosteroid insensitivel homolog prevents internode elongation and bending of the lamina joint. Plant Cell 12:1591-1606

Yan D, Zhang X, Zhang L, Ye S, Zeng L, Liu J, Li Q, He Z (2015) CURVED CHIMERIC PALEA 1 encoding an EMFT-like protein maintains epigenetic repression of OSMADS58 in rice Palea development. Plant J 82:12-24

Yin Y, Wang ZY, Mora-Garcia S, Li J, Yoshida S, Asami T, Chory J (2002) BES1 accumulates in the nucleus in response to brassinosteroids to regulate gene expression and promote stem elongation. Cell 109:181-191

Zhang B, Wang X, Zhao Z, Wang R, Huang X, Zhu Y, Yuan L, Wang Y, Xu X, Burlingame AL, Gao Y, Sun Y, Tang W (2016) OsBR/1 activates BR signaling by preventing binding between the TPR and kinase domains of OsBSK3 via phosphorylation. Plant Physiol 170:1149-1161

Zhang LY, Bai MY, Wu J, Zhu JY, Wang H, Zhang Z, Wang W, Sun Y, Zhao J, Sun X, Yang H, Xu Y, Kim SH, Fujioka S, Lin WH, Chong K, Lu T, Wang ZY (2009) Antagonistic HLH/bHLH transcription factors mediate brassinosteroid regulation of cell elongation and plant development in rice and Arabidopsis. Plant Cell 21:3767-3780

Zhang S, Wang S, XuY YC, Shen C, Qian Q, Geisler M, Jiang dA, Qi Y (2015a) The auxin response factor, OsARF19, controls rice leaf angles through positively regulating OsGH3-5 and OsBR/1. Plant Cell Environ 38:638-654

Zhang X, Sun J, Cao X, Song X (2015b) Epigenetic mutation of RAV6 affects leaf angle and seed size in rice. Plant Physiol 169:2118-2128

Zhao SQ, Hu J, Guo LB, Qian Q, Xue HW (2010) Rice leaf inclination2, a VIN3-like protein, regulates leaf angle through modulating cell division of collar. Cell Res 20:935-947

Zheng M, Wang Y, Wang Y, Wang C, Ren Y, LV J, Peng C, Wu T, Liu K, Zhao S, Liu $X$, Guo X, Jiang L, Terzaghi W, Wan JM (2015) DEFORMED FLORAL ORGAN1 (DFO1) regulates floral ORGAN identity by epigenetically repressing the expression of OsMADS58 in rice (Oryza sativa). New Phytol 206:1476-1490 